\title{
Time Evolution of a Quantum Lattice System
}

\author{
M. Pulvirenti \\ Istituto Matematico "G. Castelnuovo", Roma, Italia \\ B. Tirozzi \\ Istituto di Fisica "G. Marconi", Roma, Istituto Matematico di Camerino
}

Received October 5, 1972

\begin{abstract}
It is shown that for a quantum lattice system associated with a Hamiltonian with a kinetic part and a potential sufficiently decreasing in the particle number, the time evolution can be described, under certain assumptions, by automorphisms of a suitable algebra.
\end{abstract}

\section{Introduction}

It has been shown by Robinson [1] that the time evolution induced by the following quantum lattice system Hamiltonian:

with

$$
H_{A}=\sum_{X \subset A} \Phi(X)
$$

$$
\sum_{X \ni 0}\|\Phi(X)\| \exp (|X|-1)<+\infty
$$

where $|X|$ is the number of sites in the region $X$, can be described by an automorphism of the quasi-local algebra associated with the lattice system.

Attempts to generalize this result to a wider class of potentials have been made afterwards. Ruskai [2] has shown that for a certain class of potentials, the time evolution is at least an automorphism of an algebra larger than the observables' algebra. The case of continuous systems is not yet clear. Ruelle [3] has proven the existence of the infinite volume Green functions for low density quantum gas.

Our purpose is to consider a lattice system described by a Hamiltonian not verifying (1.1) and to show that the time evolution is an automorphism of the weak closure of the observables' algebra in the G.N.S. representation generated by an equilibrium state at low density.

In Section 2 we combine the technique of [4] and [5] in order to show the existence of the reduced density matrices (R.D.M.) at infinite volume using Ginibre's method based on the Kirkwood-Salzburg integral equations. 
In Section 3 we consider the expectation values of the time evolved local elements, and we find bounds for these quantities. On the basis of these inequalities we show the existence of the infinite volume limit of some expectation values.

In Section 4 inequalities of Section 3 will there be used in the proof of the main theorem which essentially asserts that, for small enough values of the activity and the inverse of temperature, the time evolution is regular enough to be naturally described through an algebra which is smaller than the algebra used in Ref. [2] and which has been already suggested in Ref. [6].

The straightforward but tedious estimates leading to the results of Sections 2 and 3 are developed in two Appendices.

\section{Infinite Volume R.D.M.}

Let $Z^{v}$ be a $v$-dimensional cubic lattice. Suppose that to each $\mathrm{x} \in \mathrm{Z}^{v}$ is associated a system of two operators $a_{x}, a_{x}^{+}$such that:

$$
a_{x} a_{x}^{+}+a_{x}^{+} a_{x}=1, \quad a_{x} a_{y}^{+}-a_{y}^{+} a_{x}=0(x \neq y) .
$$

We consider a system in a finite region $\Lambda \subset Z^{v}$ and assume the Hamiltonian of the system to be:

Where:

$$
H_{A}=\sum_{\substack{x, y \in A \\ x \neq y}} K(x-y) a_{x}^{+} a_{y}+\sum_{X \subset A} J(X) a^{+}(X) a(X) .
$$

$$
a^{+}(X)=\prod_{x \in X} a_{x}^{+}, \quad a^{-}(Y)=\prod_{y \in Y} a_{y}
$$

and the potentials $K(x-y), J(X)$ satisfy the following condition:

$$
\|J\| \equiv \sum_{0 \in X}|J(X)|<+\infty, \quad\|K\| \equiv \sum_{x \neq 0}|K(x)|<+\infty .
$$

Let $\mathfrak{S}_{A}$ be the Hilbert space of the vacuum representation of 2.1 .

We define $\mathfrak{A}(\Lambda)=\mathscr{B}\left(\mathfrak{H}_{A}\right)$, i.e. the algebra of bounded operators on $\mathfrak{H}_{\Lambda}$. $\tilde{\mathfrak{A}}=\bigcup_{\Lambda \subset Z^{\mathfrak{v}}} \mathfrak{A}(\Lambda)$ is a normed algebra that will be called the local algebra.

Its completion $\mathfrak{A}$ will be called the quasi-local algebra.

$\mathfrak{U}$ endowed with the natural definition of the involution, will be a $C^{*}$-algebra. It is easy to verify the following properties:

i) $\mathfrak{A}\left(\Lambda_{1}\right) \supset \mathfrak{A}\left(\Lambda_{2}\right)$ if $\Lambda_{1} \supset \Lambda_{2}$ (Isotony).

ii) $\mathfrak{A}\left(\Lambda_{1}\right)$ commutes with $\mathfrak{A}\left(\Lambda_{2}\right)$ if $\Lambda_{1} \cap \Lambda_{2}=\phi$.

iii) a translation $x \in Z^{v}$ can be represented by an automorphism $\tau_{x}$ of $Z^{v}$, such that:

$$
\tau_{x} \mathfrak{H}(\Lambda)=\mathfrak{U}(\Lambda+x)
$$


The finite volume R.D.M. are defined by:

$$
\varrho_{\Lambda}(X, Y)=\frac{\operatorname{Tr}\left(e^{-\beta H_{\Lambda}} a^{+}(X) a^{-}(Y)\right)}{\operatorname{Tr} e^{-\beta H_{\Lambda}}}
$$

Using the techniques of [4] one can show that the infinite volume limit of the R.D.M. exists:

$$
\lim _{\Lambda \rightarrow \infty} \varrho_{\Lambda}(X, Y)=\varrho(X, Y)
$$

(In the sense that $\Lambda$ eventually contains any bounded region.) Furthermore the R.D.M. satisfy the following inequality for all $A$.

$$
\left|\varrho_{\Lambda}(X, Y)\right| \leqq \text { const. } \xi^{|X|} \delta_{|X|,|Y|}
$$

where $\xi$ is a real number that can be made as small as desired by choosing $\beta$ and $z$ small enough. See Appendix I.

The numbers $\varrho_{\Lambda}(X, Y)$ define a state $\varrho_{\Lambda}$ over the algebra $\mathfrak{U}(\Lambda)$ through the formula:

$$
\varrho_{\Lambda}\left(a^{+}(X) a^{-}(Y)\right)=\varrho_{\Lambda}(X, Y), \quad X, Y \subset \Lambda
$$

and its linear extension to $\mathfrak{U}(\Lambda)$. Similarly $\varrho(X, Y)$ define a state $\varrho$ over $\tilde{\mathfrak{U}}$ through

$$
\varrho\left(a^{+}(X) a^{-}(Y)\right)=\varrho(X, Y)
$$

and its linear extension. Finally $\varrho$ can be extended by continuity to the whole of $\mathfrak{A}$. In the following we shall often write $\varrho_{A}(A)$ with $A \in \mathfrak{U}$ : this symbol makes sense if it is used when $A \in \mathfrak{U}(\bar{\Lambda})$ with $\bar{\Lambda} \subset A$ by the isotony property.

\section{Inequalities and Expectation Values of Evolving Operators}

Let $\varrho$ be the infinite volume R.D.M., $A, B, C$, the following local elements:

$$
\begin{aligned}
& A=a^{+}(X) a^{-}(Y), \\
& B=a^{+}\left(X_{1}\right) a^{-}\left(Y_{1}\right), \\
& C=a^{+}\left(X_{2}\right) a^{-}\left(Y_{2}\right)
\end{aligned}
$$

where $X, Y, X_{1}, X_{1}, X_{2}, X_{2}$ are bounded regions.

Define the following function on the finite sets $X$ contained in $\mathrm{Z}^{v}$ :

and put:

$$
M(X)=\left(4 e^{2} \xi\right)^{|X|}
$$

$$
[B, A]^{n}=\left[B,[B, A]^{n-1}\right], \text { with } \quad[B, A]^{0}=A \text {. }
$$


On the basis of inequality (2.4), we are able to prove the following:

Proposition 3.1. If $m, n$ are integers, $v=\|J\|+\|K\|$, and if the above notation is used, the following inequalities hold:

$$
\begin{array}{r}
\left|\varrho\left(B\left[H_{A}, A\right]^{n} C\right)\right| \leqq\left(2 \xi^{-1}\right)^{\left|X_{1}\right|+\left|X_{2}\right|} \frac{M(X)}{1-8 e^{2} \xi} \frac{n+1}{2} n !\left(16 v+8 e^{2} \xi\right)^{n} \\
\quad \text { for } \xi<\frac{1}{8 e^{2}}, \\
\left|\varrho\left(\left[H_{\Lambda_{1}}, B\right]^{n} A\left[H_{A_{2}}, C\right]^{m}\right)\right| \\
\leqq 2^{|X|} \frac{M\left(X_{1}\right) M\left(X_{2}\right)}{\left(1-16 e^{2} \sqrt{\xi}\right)^{2}} \frac{n+1}{2} n ! \frac{m+1}{2} m !\left(16 v+16 e^{2} \sqrt{\xi}\right)^{m+n} \\
\text { for } \sqrt{\xi}<\frac{1}{16 e^{2}} .
\end{array}
$$

For the proof see the Appendix II.

Proposition 3.2. If $\xi<\frac{1}{64 e^{4}}$ the following limit exists:

$$
\lim _{\Lambda \rightarrow \infty} \varrho\left(B\left[H_{\Lambda}, A\right]^{n} C\right) \forall n
$$

furthermore:

$$
\begin{aligned}
\lim _{\Lambda_{1} \rightarrow \infty} \lim _{\Lambda_{2} \rightarrow \infty} \varrho\left(\left[H_{\Lambda_{1}}, B\right]^{n} A\left[H_{\Lambda_{2}}, C\right]^{m}\right) \\
\quad=\lim _{\Lambda \rightarrow \infty} \varrho\left(\left[H_{\Lambda}, B\right]^{n} A\left[H_{\Lambda}, C\right]^{m}\right) \forall n, m .
\end{aligned}
$$

Proof. The existence of limit 3.1 and the equality (3.2), is implicitly proved in the Appendix II, for which the inequality (2.4) is of crucial importance.

On the basis of the preceding two propositions and the following expansion:

$$
\varrho\left(A B_{t}^{A} C\right)=\sum_{n \geqq 0} \frac{(i t)^{n}}{n !} \varrho\left(A\left[H_{A}, B\right]^{n} C\right), \quad B_{t}^{A}=e^{i H_{A} t} B e^{-i H_{A} t}
$$

the next proposition follows:

Proposition 3.3. If $t_{0}=\frac{1}{16 v+8 e^{2} \xi}, \xi<\frac{1}{64 e^{4}}$

If

$$
\lim \varrho\left(A B_{t}^{\Lambda} C\right) \quad \text { exists for } \quad|t|<t_{0} .
$$

we have:

$$
t_{0}^{\prime}=\frac{1}{16 v+16 e^{2} \sqrt{\xi}}|t|, \quad\left|t^{\prime}\right|<t_{0}^{\prime}, \quad \xi<\frac{1}{64 e^{4}},
$$

$$
\lim _{\Lambda_{1} \rightarrow \infty} \lim _{\Lambda_{2} \rightarrow \infty} \varrho\left(B_{t}^{\Lambda_{1}} A C_{t^{\prime}}^{\Lambda^{2}}\right)=\lim _{\Lambda \rightarrow \infty} \varrho\left(B_{t}^{A} A C_{t^{\prime}}^{A}\right) \text {. }
$$


If

we have:

$$
|t|<t_{0}, \quad\left|t^{\prime}\right|, \quad\left|t^{\prime \prime}\right|<t_{0}^{\prime}, \quad \xi<\frac{1}{64 e^{4}},
$$

$$
\begin{gathered}
\lim _{\Lambda \rightarrow \infty} \varrho\left(B A_{t}^{\Lambda} C\right)=\lim _{\Lambda \rightarrow \infty} \varrho_{A}\left(B A_{t}^{A} C\right) \\
\lim _{\Lambda \rightarrow \infty} \varrho\left(B_{t^{\prime \prime}}^{A} A C_{t^{\prime}}^{\Lambda}\right)=\lim _{\Lambda \rightarrow \infty} \varrho_{\Lambda}\left(B_{t^{\prime \prime}}^{A} A C_{t^{\prime}}^{\Lambda}\right) .
\end{gathered}
$$

Remark 1. The above limits exist also for complex $t, t^{\prime}, t^{\prime \prime}$, verifying the appropriate inequalities, and define analytic functions on the corresponding values.

Remark 2. It does not seem easy to prove the existence of the above limits in the norm topology sense and, actually, the limits above may well fail to exist in such a stronger sense.

\section{Existence of the Time Automorphism}

On the basis of the preceding section we are able to show the following:

Theorem 4.1. Let $\mathfrak{A}$ be the observables' algebra and $\varrho$ the state on $\mathfrak{A}$ induced by the R.D.M. Let $(\mathfrak{H}, \pi, \Omega)$ be the G.N.S. triple generated by $\varrho$ and $\pi(\mathfrak{Q})^{-}$the weak closure of $\pi(\mathfrak{Q})$.

We have, for sufficiently high values of temperature and small values of activity:

i) on $\pi(\mathfrak{H})^{-}$is defined a one parameter group of automorphisms:

$$
\alpha_{t}: \mathbb{R}^{1} \rightarrow \operatorname{aut}\left(\pi(\mathfrak{A})^{-}\right) .
$$

ii) on local elements such automorphisms are given by:

$$
\lim _{A \rightarrow \infty}\left(\Psi, \pi\left(A_{t}^{\Lambda}\right) \Phi\right)=\left(\Psi, \alpha_{t}(A) \Phi\right) \Psi, \Phi \in \mathfrak{H} .
$$

iii) there is a self-adjoint operator that generates $\alpha_{t}$.

Proof. Fixed $A \in \mathfrak{A}\left(\Lambda_{0}\right), \Lambda \supset \Lambda_{0}$ and fixed $B, C \in \mathfrak{A},|t|<t_{0}$ it follows from proposition (3.2) that the following limit exists:

$$
\lim _{\Lambda \rightarrow \infty}\left(\pi(B) \Omega, \pi\left(A_{t}^{A}\right) \pi(O) \Omega\right)=\lim _{\Lambda \rightarrow \infty} \varrho\left(B^{*} A_{t}^{\Lambda} O\right) .
$$

This limit induces a sesquilinear form $\langle\Phi, \Psi\rangle$ densely defined on $\pi(\tilde{\mathfrak{U}}) \Omega$. Furthermore, in consequence of $\left\|\pi\left(A_{t}^{\Lambda}\right)\right\| \leqq\|A\|$, this form is bounded:

$$
|\langle\Phi, \Psi\rangle| \leqq\|A\|\|\Psi\|\|\Phi\|
$$

and by density, the limit

$$
\lim _{\Lambda \rightarrow \infty}\left(\Psi, \pi\left(A_{t}^{\Lambda}\right) \Phi\right)
$$


exists for each $\Psi, \Phi \in \mathfrak{H}$. Therefore the extension of the form $\langle\Phi, \Psi\rangle$ to $\mathfrak{H} \times \mathfrak{S}$ induces a bounded operator $\tilde{\alpha}_{t}(A)$ defined by

$$
\left(\Psi, \tilde{\alpha}_{t}(A) \Phi\right)=\lim _{\Lambda \rightarrow \infty}\left(\Psi, \pi\left(A_{t}^{\Lambda}\right) \Phi\right)
$$

with $A \in \tilde{\mathfrak{A}} ; \Psi, \Phi \in \mathfrak{H} . \tilde{\alpha}_{t}(A) \in \pi(\mathfrak{A})^{-}$by construction. On the basis of proposition (3.3) we have:

$$
\begin{aligned}
\left(\Omega, \pi(B) \tilde{\alpha}_{t} \pi(C) \Omega\right) & =\lim _{A \rightarrow \infty} \varrho_{A}\left(B A_{t}^{A} C\right), \\
\left(\Omega, \tilde{\alpha}_{t}(A) \pi(B) \tilde{\alpha}_{t^{\prime}}(C) \Omega\right) & =\lim _{\Lambda \rightarrow \infty} \varrho_{\Lambda}\left(A_{t}^{A} B C_{t^{\prime}}^{A}\right) .
\end{aligned}
$$

The operator defined by $\left(|t|<t_{0}\right)$

$$
\pi(A) \Omega \rightarrow \tilde{\alpha}_{t}(A) \Omega, \quad A \in \tilde{\mathfrak{U}}
$$

is isometric because, using 4.2

$$
\begin{aligned}
\left|\tilde{\alpha}_{t}(A) \Omega\right|^{2} & =\left(\Omega, \tilde{\alpha}_{t}\left(A^{*}\right) \tilde{\alpha}_{t}(A) \Omega\right) \\
& =\lim _{A} \varrho_{\Lambda}\left(A_{t}^{* A} A_{t}^{\Lambda}\right)=\varrho\left(A^{*} A\right) \\
& =\left(\Omega, \pi\left(A^{*} A\right) \Omega\right)=|\pi(A) \Omega|^{2} .
\end{aligned}
$$

Furthermore the above operator is densely defined. So it can be extended to an operator $U_{t}$ on $\mathfrak{S}$ and therefore: $U_{t} U_{t}^{*}=I$. Furthermore $U_{t}$ is unitary because (4.1) $(B, C, A, \in \tilde{\mathfrak{A}})$ implies:

$$
\begin{aligned}
\left(\pi(B) \Omega, O_{t} \pi(A) \Omega\right) & =\lim _{A} \varrho_{\Lambda}\left(B^{*} A_{t}^{A}\right)=\lim _{A} \varrho_{A}\left(B_{-t}^{* A} A\right) \\
& =\left(\alpha_{-t}(B) \Omega, \pi(A) \Omega\right)=\left(O_{-t} \pi(B) \Omega, \pi(A) \Omega\right)
\end{aligned}
$$

which implies $U_{t}^{*}=U_{-t}$, hence $I=U_{-t} U_{-t}^{*}=U_{t}^{*} U_{t}$. By (4.1) and (4.2) and for $|t|,\left|t^{\prime}\right|<t_{0}^{\prime},\left|t+t^{\prime}\right|<t_{0}$

$$
\begin{aligned}
\left(\pi(B) \Omega, U_{t} U_{t^{\prime}} \pi(A) \Omega\right) & =\left(O_{t}^{*} \pi(B) \Omega, U_{t^{\prime}} \pi(A) \Omega\right) \\
& =\lim _{A} \varrho_{A}\left(B_{-t}^{* A} A_{t^{\prime}}^{A}\right)=\lim _{A} \varrho_{A}\left(B^{*} A_{t+t^{\prime}}^{A}\right) \\
& =\left(\pi(B) \Omega, U_{t+t^{\prime}} \pi(A) \Omega\right) .
\end{aligned}
$$

Thus $U_{t} U_{t^{\prime}}=U_{t+t^{\prime}}$.

For all $t$ let be $t=\sum_{i} t_{i},\left|t_{i}\right|<t_{0}$ we define $U_{t}=\prod_{i} U_{i}$. One can verify that $U_{t}$ does not depend on the time decomposition choosen. The mapping:

$$
\mathbb{R}^{1} \ni t \rightarrow U_{t}
$$

is a representation of the translation group implemented by unitary operators. The mapping:

$$
\mathbb{R}^{1} \ni t \rightarrow \alpha_{t} \quad \text { where } \quad \alpha_{t}(A)=U_{t} A U_{t}^{-1}, \quad A \in \pi(\mathfrak{H})^{-}
$$


is a representation of $\mathbb{R}^{1}$ by automorphisms of the Von Neumann algebra $\pi(\mathfrak{A})^{-}$. (4.1) and (4.2) imply $\alpha_{t}$ is an extension of $\tilde{\alpha}_{t}$ :

(One can easily verify that the automorphism $A \rightarrow U_{t} A U_{t}^{-1}$ is weakly continuous so $U_{t} A U_{t}^{-1} \in \pi(\mathfrak{H})^{-}$if $\left.A \in \pi(\mathfrak{H})^{-}\right)$

$$
\begin{aligned}
\left(\pi(B) \Omega, U_{t} \pi(A) U_{t}^{-1} \pi(C) \Omega\right) & =\left(U_{-t} \pi(B) \Omega, \pi(A) U_{-t} \pi(C) \Omega\right) \\
& =\lim _{A} \varrho_{A}\left(B_{-t}^{* A} A C_{-t}^{A}\right) \\
& =\lim _{A} \varrho_{A}\left(B^{*} A_{t}^{A} C\right)=\left(\pi(B) \Omega, \tilde{\alpha}_{t}(A) \pi(C) \Omega\right)
\end{aligned}
$$

Finally by the analyticity (for $|t|<t_{0}$ ) of the functions:

$$
t \rightarrow\left(\pi(A) \Omega, \alpha_{t}(A) \Omega\right) \quad A \in \tilde{\mathfrak{U}}
$$

(see Remark 1 at the end of Section 3) $U_{t}$ is strongly continuous at the origin and thus there exists a self-adjoint operator $H_{Q}$ such that:

$$
U_{t}=e^{i H_{Q} t} .
$$

Remark 1. We observe that the proof of theorem 4.1, is not very satisfactory since we have essentially used the inequality:

$$
\left\lfloor(X, Y) \mid \leqq C \xi^{|X|} \quad \xi<\frac{1}{64 e^{4}}\right.
$$

while it would be more satisfactory to have a proof based on:

$$
\varrho(X, Y) \mid \leqq C \eta^{|X|} \quad \eta<1
$$

which is expected to hold for the equilibrium states at all densities and temperatures.

Remark 2. We were not able to make use of the theorem in Ref. [6] because it requires the convergence of the expectation values of the product of many evolved operators. This is impossible to demonstrate in our model. On the contrary, our Theorem 4.1 requires the convergence of the expectation values of the product of few evolved operators.

\section{Appendix I}

We use the notation and definition of [4]. Define the mean potential energy of a set of simple trajectories as

We put:

$$
J\left(\omega_{1} \ldots \omega_{n}\right)=\int_{0}^{1} J\left(\omega_{1}(t) \ldots \omega_{n}(t)\right) d t .
$$

$$
J^{\prime}(\boldsymbol{\eta})=\sum_{\substack{\phi \neq \tilde{e}_{1} \subset \tilde{\eta}_{1} \\ \dot{\phi} \neq \tilde{\varrho}_{k} \subset \tilde{\eta}_{k}}} J\left(\tilde{\varrho}_{1}, \ldots, \tilde{\varrho}_{k}\right)
$$


where generally $\tilde{\omega}$ is a composite trajectory $(l(\tilde{\omega}) \geqq 1)$ and $\tilde{\varrho} \subset \tilde{\eta}$ means that $\tilde{\varrho}$ is a subset of simple trajectory contained in the composite $\tilde{\eta}$. Furthermore the notation $J\left(\tilde{\varrho}_{1} \ldots \tilde{\varrho}_{k}\right)$ means:

$$
J\left(\tilde{\varrho}_{1} \ldots \tilde{\varrho}_{k}\right)=J\left(\varrho_{11} \varrho_{12} \ldots \varrho_{k n_{k}}\right)
$$

where the subset $\varrho_{1}$ is the subset $\left\{\varrho_{11}, \varrho_{12} \ldots \varrho_{1 k_{1}}\right\}$ and all $\varrho_{i j}$ are simple.

Define:

one finds:

$$
U(\omega)=\sum_{\eta \subset \omega} J^{\prime}(\eta)
$$

and

$$
Z_{\Lambda}=\operatorname{Tr} e^{-\beta H_{A}}=\sum_{X \subset A} \int_{\omega \subset A}^{\prime} \tilde{P}_{X, Y}(d \omega) e^{-\beta U(\omega)}
$$

$$
\varrho_{A}(X, Y)=\int \tilde{P}_{X, Y}(d \omega) \varrho_{\Lambda}(\omega) .
$$

Where $\hat{\varrho}_{A}(\omega)$ is defined by:

$$
\hat{\varrho}_{\Lambda}(\omega)=\frac{1}{Z_{\Lambda}} \int_{\omega^{\prime} \subset A} d \omega^{\prime} e^{-\beta U\left(\omega \cup \omega^{\prime}\right)}
$$

Let $\omega_{1}$ the first simple trajectory of $\omega$ (choosen in the lexicographic order) and $\omega^{(1)}$ the set of trajectories obtained by subtracting $\omega_{1}$ from $\omega$, we have:

where

$$
\begin{aligned}
U\left(\omega \cup \omega^{\prime}\right)= & U\left(\omega^{(1)} \cup \omega^{\prime}\right)+\sum_{\omega_{1} \in \eta \subset \omega} J^{\prime}(\boldsymbol{\eta}) \\
& +\sum_{\phi \neq \gamma \subset \omega^{\prime}} W(\omega, \gamma)
\end{aligned}
$$

$$
W(\omega, \gamma)=\sum_{\omega_{1} \in \eta \subset \omega} J^{\prime}(\eta \cup \gamma) .
$$

Putting (A.I.2) in (A.I.1) we obtain the same integral equation of [4] for $\varrho_{A}$ with the following integral kernel:

$$
\begin{aligned}
K^{(1)}(\boldsymbol{\omega}, \boldsymbol{\eta}) & =1 \quad \boldsymbol{\eta}=0 \\
& =0 \quad \boldsymbol{\eta} \cap \omega_{1} \neq \phi \\
& =\sum_{n \geqq 1} \sum_{\left\{\gamma_{1} \ldots \gamma_{n}\right\} U_{l} \gamma_{l}=\eta} \prod_{i=1}^{n}\left(e^{-W\left(\omega, \gamma_{l}\right)}-1\right)
\end{aligned}
$$

if $\boldsymbol{\eta} \cap \omega_{1}=\phi$ and $\boldsymbol{\eta} \neq \phi$.

Let us introduce the Banach space $\mathscr{E}_{\tau}$ of all functions $\varphi$ defined on set of trajectories, which vanish on "overlapping" trajectories, such that:

$$
\|\varphi\|_{\tau}=\sup _{\omega} \frac{|\varphi(\omega)|}{\tau^{n(\omega)}} \tau>1
$$


In such a space the integral equation becomes:

where

$$
\hat{\varrho}_{A}=z \chi_{A} \alpha+z \chi_{A} e^{-\beta U^{(1)}} \mathscr{K} \hat{\varrho}_{\Lambda}
$$

and

where

$$
\mathscr{K}=E+F+G \quad(\text { see }[4])
$$

and

$$
\left(e^{-\beta U^{(1)}} \varphi\right)(\boldsymbol{\omega})=e^{-\beta U^{(1)}(\boldsymbol{\omega})} \varphi(\boldsymbol{\omega})
$$

$$
U^{(1)}(\boldsymbol{\omega})=\sum_{\substack{\omega_{1} \in \boldsymbol{\eta} \subset \boldsymbol{\omega} \\ \boldsymbol{\eta} \neq\left\{\omega_{1}\right\}}} J^{\prime}(\boldsymbol{\eta})
$$

$$
Z=e^{\beta \mu}, \quad \mu=-J(\{x\}) .
$$

The existence of infinite volume R.D.M. follows from the existence of classical correlation functions $\varrho(\omega)$ in the same limit. Putting

we have

$$
\mathscr{H}=e^{-\beta U^{(1)}} \mathscr{K}
$$

and

where

$$
\|\mathscr{H}\|_{\tau} \leqq e^{\beta\|J\|}\|\mathscr{K}\|_{\tau}
$$

$$
\|\mathscr{K}\|_{\tau} \leqq\|E\|_{\tau}+\|F\|_{\tau}+\|G\|_{\tau}
$$

$\|E\|_{\tau} \leqq e^{\lambda}, \quad \lambda=-\frac{\log \left(1+2 \tau \beta\|K\| e^{1-2 \beta K}\right)}{2 \tau \beta\|K\|}+\frac{e^{1-2 \beta K}}{1-2 \tau \beta\|K\| e^{1-2 \beta K}}$

$\|G\|_{\tau} \leqq\left(1+e^{\lambda}\right) \mid \exp \left[\left|e^{\beta\|\nu\|}-1\right|\left(\left(1-2 \beta \tau\|K\| e^{1-2 \beta K}\right)^{\frac{1}{2 \beta \tau}\|K\|}-1|-1|\right.\right.$

$\|F\|_{\tau} \leqq 1$.

And these estimates are valid under the following conditions:

$$
2 \beta \tau\|K\|<1, \quad 2 \beta \tau\|K\| e^{1-2 \beta K}<1, \quad e^{\lambda}<\tau .
$$

We have the existence of infinite volume R.D.M. for those values of $z$ and $\beta$ for which $|z|\|\mathscr{H}\|_{\tau}<1$. We have used the same norm as in Ref. [4] in the case $\sigma=1$. This is necessary in order to obtain the estimates (A.I.4), which cannot be obtained for our Hamiltonian with $\sigma<1$.

Furthermore our estimates are a refinement of the ones in Ref. [4]. In fact in order to avoid overlapping we have imposed explicitly the condition $n \geqq j-1$ for a trajectory of length $j$ with $n$ jumps. In this way we obtain the inequality (2.4)

$$
\begin{aligned}
|\varrho(X, Y)| \leqq & \int\left|\tilde{P}_{X Y}(d \omega)\right||\hat{\varrho}(\omega)| \\
\leqq & |z| \sum_{n \geqq|X|-1}\left(|z|\|\mathscr{H}\|_{\tau}\right)^{n} \int\left|\tilde{P}_{X Y}(d \omega)\right| \tau^{n(\omega)} \\
\leqq & |z| \sum_{n \geqq|X|-1}\left(|z|\|\mathscr{H}\|_{\tau}\right)^{n} \\
& \times\left|\sum_{J_{1} \ldots J_{K}} \sum_{\mathscr{P}} \int P_{x_{1} \mathscr{P}_{1}(y)}^{J_{1}}\left(d \omega_{1}\right) \ldots P_{x_{K} \mathscr{P}_{K}(y)}^{J_{K}}\left(d \omega_{K}\right) \tau^{n\left(\omega_{1}\right)+\ldots n\left(\omega_{K}\right)}\right| \\
\leqq & \frac{\left(|z| \chi\|\mathscr{H}\|_{\tau}\right)^{|X|}}{\|\mathscr{H}\|_{\tau}\left(1-|z|\|\mathscr{H}\|_{\tau}\right)}
\end{aligned}
$$


where

$$
\chi=\frac{e^{1-2 \beta \tau K}}{\left(1-e^{1-2 \beta \tau K} 2 \beta \tau\|K\|\right)(1-2 \beta \tau\|K\|)}
$$

and thus (2.4) putting:

$$
\xi=|z| \chi\|\mathscr{H}\|_{\tau}
$$

\section{Appendix II}

Proof of Proposition 3.1.

By definition we have the following commutator:

$$
\begin{aligned}
{\left[H_{A}, a^{+}(X) a^{-}(Y)\right]=} & \sum_{x \in \Lambda} \sum_{y \in X} K(x-y) a^{+}\left(\frac{X}{y} \cup x\right) a(Y) \\
& +-\sum_{y \in \Lambda} \sum_{x \in Y} K(x-y) a^{+}(X) a^{-}\left(\frac{Y}{x} \cup y\right) \\
& +-2 \sum_{x \in A} \sum_{y \in X} K(x-y) a^{+}(X \cup x) a^{-}(Y \cup y) \\
& +2 \sum_{y \in \Lambda} \sum_{x \in Y} K(x-y) a^{+}(X \cup x) a^{-}(Y \cup y) \\
& +\sum_{S \neq \phi}\left\{\sum_{\phi \neq T \subset X} J(S \cup T)-\sum_{\phi \neq T \subset Y} J(S \cup T)\right\} \\
& \left.\cdot a^{+}(X \cup Y)=\phi\right) a^{-}(Y \cup S) \\
& +\sum_{T \subset X} J(T) a^{+}(X) a^{-}(Y)-\sum_{T \subset Y} J(T) a^{+}(X) a^{-}(Y)
\end{aligned}
$$
where $X$ and $Y$ are bounded regions and $\frac{X}{y}$ means the set $X$ prived of the
point $y$.

On the linear space generated by the elements: $a^{+}(X) a^{-}(Y)$, define the following operators:

$$
\begin{aligned}
\Delta^{+} a^{+}(X) a^{-}(Y)= & \sum_{\xi \in \Lambda} \sum_{x \in X} K(\xi-x) a^{+}\left(\frac{X}{x} \cup \xi\right) a^{-}(Y) \\
\Delta^{-} a^{+}(X) a^{-}(Y)= & \sum_{\eta \in A} \sum_{y \in Y} K(\eta-y) a^{+}(X) a^{-}\left(\frac{Y}{y} \cup \eta\right) \\
U^{+} a^{+}(X) a^{-}(Y)= & U(X) a^{+}(X) a^{-}(Y) \\
U^{-} a^{+}(X) a^{-}(Y)= & U(Y) a^{+}(X) a^{-}(Y) \\
\mathscr{B}_{1} a^{+}(X) a^{-}(Y)= & \sum_{\substack{S \neq \phi \\
S \cap(X \cup Y)=\phi}}[w(X, S)-w(Y, S)] \\
& \cdot a^{+}(X \cup S) a^{-}(Y \cup S)
\end{aligned}
$$


where

$$
W(X, S)=\sum_{\phi \neq T \subset X} J(S \cup T)
$$

$\mathscr{B}_{2} a^{+}(X) a^{-}(Y)=2 \sum_{S, S^{\prime}}\left[W_{X}^{(2)}\left(S^{\prime}, S\right)-W_{Y}^{(2)}\left(S, S^{\prime}\right)\right] a^{+}(X \cup S) \cdot a^{-}(Y \cup S)$

where:

$W_{Z}^{(2)}\left(S^{\prime}, S\right)=-K\left(S-S^{\prime}\right) \quad$ if $S^{\prime}, S$ are points $S \cap Z=\phi S^{\prime} \subset Z$ $=0$ otherwise.

Putting:

$$
\begin{aligned}
W_{Z}^{(1)}\left(S, S^{\prime}\right) & =\frac{1}{2} W(Z, S) \quad \text { if } \quad S \equiv S^{\prime}, S \neq \phi, S \cap Z=\phi \\
& =0 \quad \text { otherwise, } \\
W_{Z}\left(S, S^{\prime}\right) & =W_{Z}^{(1)}\left(S, S^{\prime}\right)+W_{Z}^{(2)}\left(S, S^{\prime}\right)
\end{aligned}
$$

one has:

$$
\begin{gathered}
\mathscr{B}=\mathscr{B}_{1}+\mathscr{B}_{2} \\
\mathscr{B} a^{+}(X) a^{-}(Y) \quad \\
=2 \sum_{S, S^{\prime}}\left[W_{X}\left(S, S^{\prime}\right)-W_{Y}\left(S^{\prime}, S\right)\right] a^{+}(X \cup S) a^{-}(Y \cup S) .
\end{gathered}
$$

Finally putting:

$$
\mathscr{H}^{+}=\Delta^{+}+U^{+} \quad \mathscr{H}^{-}=\Delta^{-}+U^{-} \quad \mathscr{A}=\mathscr{H}^{+}-\mathscr{H}^{-}
$$

one has for (A.II.1) and (A.II.8)

$$
\begin{aligned}
{\left[H_{A}, a^{+}(X) a^{-}(Y)\right] } & =(\mathscr{A}+\mathscr{B}) a^{+}(X) a^{-}(Y) \\
{\left[H_{\Lambda}, a^{+}(X) a^{-}(Y)\right]^{n} } & =(\mathscr{A}+\mathscr{B})^{n} a^{+}(X) a^{-}(Y)
\end{aligned}
$$

with the following expansion:

$$
\begin{aligned}
& (\mathscr{A}+\mathscr{B})^{n} \\
& =\sum_{k=0}^{n} \sum_{r=1}^{\left[\frac{n+1}{2}\right]} \sum_{\substack{h_{1} \ldots h_{r}, k_{1} \ldots k_{r}: \\
i=1, \ldots, r}}\left(\mathscr{A}^{h_{1}} \mathscr{B}^{k_{1}} \ldots \mathscr{A}^{h_{r}} \mathscr{B}^{k_{r}}+\mathscr{B}^{h_{1}} \mathscr{A}^{k_{1}} \ldots \mathscr{B}^{h_{r}} \mathscr{A}^{k_{r}}\right) .
\end{aligned}
$$


We shall transform (A.II.9) into a more handable form.

$$
\begin{aligned}
\mathscr{B}^{K_{r}} a^{+}(X) a^{-}(Y)= & 2^{K_{r} \mathscr{B}^{K_{r}-1}} \\
& \cdot \sum_{T, T^{\prime}}\left[W_{X}\left(T, T^{\prime}\right)-W_{Y}\left(T, T^{\prime}\right)\right] a^{+}(X \cup T) a^{-}\left(Y \cup T^{\prime}\right) \\
= & 2^{K_{r}} \sum_{\substack{T_{1} \ldots T_{K_{r}} \\
T_{1}^{\prime} \ldots T_{K_{r}}}}\left(\prod _ { i = 1 } ^ { K _ { r } } \left[W_{X \cup T_{1} \ldots T_{J-1}}\left(T_{j}, T_{j}^{\prime}\right)\right.\right. \\
& \left.+-\underset{Y}{-W_{Y \cup T_{1}^{\prime} \ldots T_{j-1}^{\prime}}}\left(T_{j}^{\prime}, T_{j}\right)\right] a^{+}\left(X \cup T_{\left.1 \cup \ldots \cup T_{k_{r}}\right)}\right) \\
& \cdot a^{-}\left(Y \cup T_{1}^{\prime} \cup \ldots \cup T_{k_{r}}^{\prime}\right) \\
= & \sum_{S, S^{\prime}} F_{k_{r}}\left(X, Y \mid S, S^{\prime}\right) a^{+}(X \cup S) a^{-}\left(Y \cup S^{\prime}\right)
\end{aligned}
$$

where:

$$
\begin{aligned}
& F_{k_{r}}\left(X, Y \mid S, S^{\prime}\right) \\
& =2^{k_{r}} \sum_{\substack{T_{1} \ldots T_{k_{r}} \\
T_{1} \ldots T_{k_{r}} \\
U_{2} T_{i}=S \\
U_{\imath} T_{l}^{\prime}=S^{\prime}}} \prod_{j=1}\left[W_{X \cup T_{1} \ldots T_{J-1}}\left(T_{j}, T_{j}^{\prime}\right)-W_{Y \cup T_{1}^{\prime} \ldots T_{J-1}^{\prime}}\left(T_{j}^{\prime}, T_{j}\right)\right] .
\end{aligned}
$$

Further we describe the action of $\mathscr{A}^{h_{r}}$ with the aid of certain coefficient $E_{h_{r}}\left(P, Q ; P^{\prime}, Q^{\prime} \mid \cdot\right)$ :

$$
\mathscr{A}^{h_{r}} a^{+}(X) a^{-}(Y)=\sum_{\substack{P, Q \\ P^{\prime}, Q^{\prime}}} E_{h_{r}}\left(P, Q ; P^{\prime}, Q^{\prime} \mid X, Y\right) a^{+}\left(\frac{X \cup P}{Q}\right) a^{-}\left(\frac{Y \cup P^{\prime}}{Q^{\prime}}\right)
$$

where the summation on $P, Q, P^{\prime}, Q^{\prime}$ verifies the required compatibility conditions, i.e.

$$
\begin{array}{ll}
P \cap X=\phi, & Q \subset X \cup P, \\
P^{\prime} \cap Y=\phi, & Q^{\prime} \subset Y \cup P^{\prime}, \quad P, P^{\prime}, Q, Q^{\prime} \subset \Lambda .
\end{array}
$$

For the $n^{\text {th }}$ commutator, we write:

$$
\left[H_{\Lambda}, a^{+}(X) a^{-}(Y)\right]^{n}=\sum_{\tau} C_{\tau}^{(n)} a^{+}\left(X_{\tau}\right) a^{-}\left(Y_{\tau}\right)
$$

where the coefficients $C_{\tau}^{(n)}$ depend on the quantities introduced in A.II.10 and 11. Now let $\tilde{X}_{1}, \tilde{Y}_{1}, \tilde{X}_{2}, \tilde{Y}_{2}$, be bounded regions contained in $\Lambda$ $B=a^{+}\left(\tilde{X}_{1}\right) a^{-}\left(\tilde{Y}_{1}\right) C=a^{+}\left(\tilde{X}_{1}\right) a^{-}\left(\tilde{Y}_{2}\right)$. One has:

$$
\left|\varrho\left(B A_{t}^{\Lambda} C\right)\right| \leqq \sum_{n \geqq 0} \frac{|t|^{h}}{n !} \sum_{\tau} C_{\tau}^{(n)}\left|\varrho\left(a^{+}\left(\tilde{X}_{1}\right) a^{-}\left(\tilde{Y}_{2}\right) a^{+}\left(X_{\tau}\right) a^{-}\left(Y_{\tau}\right) a^{+}\left(\tilde{X}_{2}\right) a^{-}\left(\tilde{Y}_{2}\right)\right)\right|
$$


and, using the commutation rules and (2.4)

$$
\begin{aligned}
& \varrho \varrho\left(a^{+}\left(\tilde{X}_{1}\right) a^{-}\left(\tilde{Y}_{1}\right) a^{+}\left(X_{\tau}\right) a^{-}\left(Y_{\tau}\right) a^{+}\left(\tilde{X}_{2}\right) a^{-}\left(\tilde{Y}_{2}\right)\right) \mid \\
&=\sum_{P \subset T} \sum_{P^{\prime} \subset S} \mid \varrho\left(a^{+}\left(\tilde{X}_{1} \cup \frac{X_{\tau}}{T} \cup P \cup \frac{\tilde{X}_{2}}{S} \cup P^{\prime}\right)\right. \\
& \\
&\left.\quad \cdot a^{-}\left(\frac{Y_{\tau} \cup \frac{\tilde{Y}_{1}}{T} \cup P}{S} \cup \tilde{Y}_{2} \cup P^{\prime}\right)\right) \mid \\
& \leqq\left(2 \xi^{-1}\right)^{\left|\tilde{X}_{2}\right|+\left|\tilde{Y}_{2}\right| \xi\left|\xi_{\tau}\right|} .
\end{aligned}
$$

Where in the second step:

Thus we have:

$$
\begin{aligned}
& \tilde{Y}_{1} \cap X_{\tau}=T \\
& \tilde{X}_{2} \cap\left(Y_{\tau} \cup \frac{\tilde{Y}_{1}}{T} \cup P\right)=S .
\end{aligned}
$$

$$
\left|\varrho\left(B A_{t}^{A} C\right)\right| \leqq\left(2^{-1} \xi\right)^{-\left|\tilde{X}_{1}\right|-\left|\tilde{X}_{2}\right|} \sum_{u \geqq 0} \frac{|t|^{u}}{u !} \sum_{\tau}\left|C_{\tau}^{(n)}\right| \xi^{\left|X_{\tau}\right|} .
$$

Now we are able to give an estimate of the sums $\sum_{\tau}\left|C_{\tau}^{(u)}\right|$ through an estimate of $F_{K_{r}}(\cdot)$ and $E_{h_{r}}(\cdot)$. We have:

$$
\begin{aligned}
& \sum_{\substack{S, S^{\prime} \\
|S|=\left|S^{\prime}\right|=\alpha}}\left|F_{k}\left(Z_{1}, Z_{2} \mid S, S^{\prime}\right)\right| \leqq 2^{k} \sum_{\substack{S, S^{\prime} \\
|S|=\left|S^{\prime}\right|=\alpha}} \sum_{\substack{T_{1} \ldots T_{k} \\
T_{1}^{\prime} \ldots l \\
U_{l} T_{k}=S \\
U_{i} T_{i}^{\prime}=S^{\prime}}} \\
& \cdot \prod_{j=1}^{k}\left|W_{Z_{1} \cup T_{1} \ldots T_{J-1}}\left(T_{j}^{\prime}, T_{j}\right)-W_{Z_{2} \cup T_{1}^{\prime} \cup \ldots \cup T_{j-1}^{\prime}}\left(T_{j}^{\prime}, T_{j}\right)\right| \\
& =2^{k} \sum_{\substack{\sigma_{1} \ldots \sigma_{k} \\
\sum_{1} \sigma_{i}=\alpha}} \sum_{\substack{T_{1} \ldots T_{k} \\
T_{1} \ldots T_{1}^{\prime} \\
\sigma_{l}=\left|T_{i}\right|=\left|T_{i}^{\prime}\right| \\
\forall i=1 \ldots k}} \prod_{j=1}^{k}\left|W_{Z_{1} \cup T_{1} \cup \cdots \cup T_{j-1}}\left(T_{j}, T_{j}^{\prime}\right)-W_{Z_{2} \cup T_{1}^{\prime} \cup \cdots \cup T_{j-1}^{\prime}}\left(T_{j}^{\prime}, T_{j}\right)\right|
\end{aligned}
$$

Where the last step is justified if we remember that the products are zero if $\left|T_{i}\right| \neq\left|T_{i}^{\prime}\right|$ and that $T_{j} \cap T_{i}=\phi \forall i, j$. (The same for $T^{\prime}$.) On the basis of (A.II.3,4,5,6),

$$
\begin{aligned}
\sum_{\substack{S, S^{\prime} \\
|S|=\left|S^{\prime}\right|=\alpha}}\left|F_{k}\left(Z_{1}, Z_{2} \mid S, S^{\prime}\right)\right| \\
\quad \leqq 4^{k} \sum_{\substack{\sigma_{1} \ldots \sigma_{k} \\
\sum \sigma_{l}=\alpha}}\left|Z_{1}\right|\left(\|J\|_{\sigma_{1}}+\|K\|\right) \ldots\left(\left|Z_{1}\right|+\alpha\right)\left(\|J\|_{\sigma_{k}}+\|K\|\right) \\
=[4 v(|z|+\alpha)]^{k} 2^{\alpha} .
\end{aligned}
$$


Where $v=\|J\|+\|K\|$ and $\|J\|_{\sigma}=\sum_{\substack{0 \in X \\|X|>\sigma}}|J(X)| \leqq\|J\|$. The first inequality
is valid because:

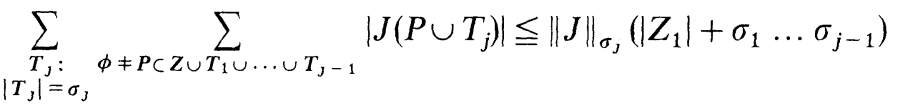

$$
\begin{aligned}
& \sum_{\substack{T_{j}, T_{j}^{\prime} \\
\left|T_{J}\right|=\left|T_{j}^{\prime}\right|=\sigma_{J}=1}}\left|K\left(T_{j}-T_{j}^{\prime}\right)\right| \leqq\|K\|\left(|Z|+\sigma_{1}+\cdots+\sigma_{j-1}\right) .
\end{aligned}
$$

Finally for

$$
\left|\sum_{P, Q, P^{\prime}, Q^{\prime}} E_{h}\left(P, Q ; P^{\prime}, Q^{\prime} \mid Z_{1}, Z_{2}\right)\right| \leqq\left(2 v\left|Z_{1}\right|\right)^{h}
$$

one has, defining the coefficients $C^{\prime}\left(\alpha_{1} \ldots \alpha_{2}\right)$ through:

$$
\sum_{\tau} C_{\tau}^{(n)} \xi^{\left|X_{\tau}\right|}=\sum_{k=0}^{n} \sum_{r=1}^{\left[\frac{n+1}{2}\right]} \sum_{\substack{h_{1} \ldots h_{r} \\ k_{1} \ldots k_{r} \\ h_{1}>0, i=1 \ldots r}} \sum_{\alpha_{1} \ldots \alpha_{r}} C^{\prime}\left(\alpha_{1} \ldots \alpha_{r}\right) \xi^{|X|+\sum_{i=1}^{r} \alpha_{l}}
$$

the following inequality:

$$
\begin{aligned}
& \sum_{\alpha_{1} \ldots \alpha_{r}} C^{\prime}\left(\alpha_{1} \ldots \alpha_{r}\right) \leqq 2 \sum_{\alpha_{1} \ldots \alpha_{r}} \prod_{j=1}^{r}\left[2 v\left(|X|+\sum_{i=1}^{j} \alpha_{i}\right)\right]^{h_{i}} . \\
& \prod_{s=1}^{r}\left[4 v\left(|X|+\sum_{j=1}^{s} \alpha_{i}\right)\right]^{k_{s}} 2^{\sum^{i \alpha_{i}}}(2 \xi)^{|X|+\sum_{i=1}^{r} \alpha_{i}} \\
& \leqq \sum_{\alpha_{1} \ldots \alpha_{2}}(4 v)^{\sum_{i} h_{i}+\sum k_{i}}\left(\sum_{i} h_{i}\right) !\left(\sum_{i} k_{i}\right) !\left(4 e^{2} \xi\right)^{|X|+\sum_{l} \alpha_{2}} \text {. }
\end{aligned}
$$

And thus:

$$
\begin{aligned}
& \sum_{\tau} C_{\tau}^{(n)} \xi^{\left|X_{\tau}\right|} \leqq \sum_{0}^{r} \sum_{0}^{\left[\frac{n+1}{2}\right]} \sum_{\substack{h_{1} \ldots h_{r}: \sum h_{i}=k \\
k_{1} \ldots k_{r}: \sum k_{l}=h-k}} \sum_{\substack{\alpha_{1} \ldots \alpha_{r} \\
\sum_{i} \geqq h-k}} \\
& h_{1}>0, i=1 \ldots r \\
& k_{1}>0, i=1 \ldots r-1 \\
& 4 v^{\sum_{i} h_{i}+\sum_{i J} k_{2}}\left(\sum_{i} h_{i}\right) !\left(\sum_{i} k_{i}\right) !\left(4 e^{2} \xi\right)^{|X|+\sum_{i} \alpha_{i}} \\
& \leqq \frac{M(X)}{1-8 e^{2} \xi} \sum_{0}^{n} k \sum_{0}^{\left[\frac{n+1}{2}\right]} \sum_{\substack{h_{1} \ldots h_{r} \\
k_{1} \cdots k_{r}}}(4 v)^{\sum h_{i}+\sum_{2} k_{2}}\left(\sum_{i} h_{i}\right) !\left(\sum_{i} k_{i}\right) !\left(8 e^{2} \xi\right)^{h-k}
\end{aligned}
$$




$$
\begin{aligned}
& \leqq \frac{M(X)}{1-8 e^{2} \xi} \sum_{0}^{n} \sum_{0}^{\left[\frac{n+1}{2}\right]}(16 v)^{n} k !(n-k) !\left(8 e^{2} \xi\right)^{h-k} \\
& \leqq \frac{M(X)}{1-8 e^{2} \xi} \frac{n+1}{2} n !\left(16 v+8 e^{2} \xi\right)^{n}
\end{aligned}
$$

for $\xi<\frac{1}{8 e^{2}}$. So the first part of Proposition 3.1 is proved.

The second part follows easily by the above estimates and by these inequalities:

$$
\begin{aligned}
& \varrho \varrho\left(\left[H_{\Lambda_{1}}, A\right]^{n} B\left[H_{\Lambda_{2}}, C\right]^{m}\right) \mid \\
& \leqq \sum_{\tau} C_{\tau}^{(n)} \sum_{\sigma} C_{\sigma}^{(m)}\left|\varrho\left(a^{+}\left(X_{1}^{\tau}\right) a^{-}\left(Y_{1}^{\tau}\right) a^{+}(X) a^{-}(Y) \cdot a^{+}\left(X_{2}^{\sigma}\right) a^{-}\left(Y_{2}^{\sigma}\right)\right)\right| \\
& \leqq \sum_{\tau} C_{\tau}^{(n)} \sum_{\sigma} C_{\sigma}^{(m)} \sum_{P \subset T} \sum_{P^{\prime} \subset S} \mid \varrho\left(a^{+}\left(X_{1}^{\tau} \cup \frac{X}{T} \cup P \cup \frac{X_{2}^{\sigma}}{S} \cup P^{\prime}\right)\right. \\
& \left.\cdot a^{-}\left(\frac{Y \cup \frac{Y_{1}^{\tau}}{T} \cup P}{S} \cup Y_{2}^{\sigma} \cup P^{\prime}\right)\right) \\
& X_{1}^{\tau} \cap X=T \\
& X_{2}^{\sigma} \cap\left(Y \cup \frac{Y_{1}^{\tau}}{T} \cup P\right)=S
\end{aligned}
$$

by putting

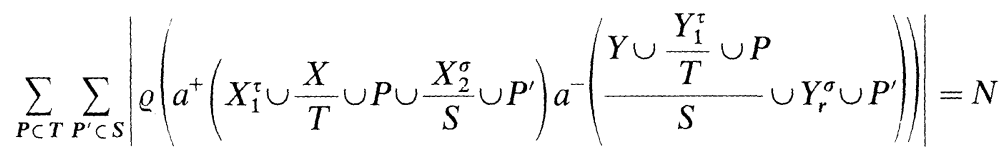

we have

but also

and hence

$$
N \leqq 2^{|X|} 2^{\left|X_{2}^{\sigma}\right|} \xi^{\left|X_{1}^{\tau}\right|}
$$$$
N \leqq 2^{|X|} 2^{\left|X_{2}^{\sigma}\right|} \xi^{\left|X_{2}^{\sigma}\right|}
$$

$$
N \leqq 2^{|X|} 2^{\left|X_{2}^{\sigma}\right|}(\sqrt{\xi})^{\left|X_{1}^{\tau}\right|+\left|X_{2}^{\sigma}\right|} \leqq 2^{|X|}(2 \sqrt{\xi})^{\left|X_{1}^{\tau}\right|+\left|X_{2}^{\sigma}\right|}
$$

Finally we have

$$
\begin{aligned}
& \varrho\left(\left[H_{\Lambda_{1}}, A\right]^{n} B\left[H_{A_{2}}, C\right]^{m}\right) \mid \\
& \leqq \sum_{\tau} C_{\tau}^{(n)} \sum_{\sigma} C_{\sigma}^{(m)} 2^{|X|}(2 \sqrt{\xi})^{\left|X_{1}^{\tau}\right|+\left|X_{2}^{\sigma}\right|} .
\end{aligned}
$$

Acknowledgments. We thank Prof. G. Gallavotti for suggesting the idea of this work and for his continuous help. We are indebted also to Prof. D. Ruelle for suggesting some ideas for the proof of Theorem 4.1 . 
Note added in proof. When this work was finished, we have received a private communication by E. B. Davies who has found a result very similar to our Theorem 4.1.

\section{References}

1. Robinson, D. W.: Commun. math. Phys. 6, 151-160 (1967); Commun. math. Phys. 7, $337-348$ (1968).

2. Ruskai, M. B.: Commun. math. Phys. 20, 193-204 (1971).

3. Ruelle, D.: J. Math. Phys. 12, 901 (1971).

4. Gallavotti, G., Miracle Sole, S., Robinson, D. W.: Commun. math. Phys. 19, 311-324 (1968).

5. Gallavotti, G., Miracle Sole, S.: Commun. math. Phys. 5, 317-323 (1967).

6. Dubin, D. A., Sewell, G.: J. Math. Phys. 11, 2990-2998 (1970).

M. Pulvirenti

Istituto Matematico

"G. Castelnuovo"

Roma, Italia 